\title{
The prevalence and clinical significance of inhalant withdrawal symptoms among a national sample
}

\author{
This article was published in the following Dove Press journal: \\ Substance Abuse and Rehabilitation \\ I April 201 I \\ Number of times this article has been viewed
}

\section{Brian E Perron' \\ Joseph E Glass ${ }^{2}$ \\ Brian K Ahmedani ${ }^{3}$ \\ Michael G Vaughn ${ }^{4}$ \\ Daniel E Roberts' \\ Li-Tzy Wu ${ }^{5}$}

'University of Michigan, Ann Arbor, MI, USA; ${ }^{2}$ Washington University, St Louis, MO, USA; ${ }^{3}$ Henry Ford Health System, Detroit, MI, USA; ${ }^{4}$ St Louis University, St Louis, MO, USA; ${ }^{5}$ Duke University, Durham, NC, USA
Correspondence: Brian E Perron School of Social Work, 1080 South University Avenue, University of Michigan, Ann Arbor, MI 48109, USA

Tel +l 7347639272

Email beperron@umich.edu
Background: Inhalants are among the most common and dangerous forms of substance use, but very little research on inhalant use disorders exist. Unlike other substances, the Diagnostic and Statistical Manual, 4th edition (DSM-IV) indicates that inhalants do not have an associated withdrawal syndrome among persons who meet criteria for inhalant dependence.

Methods: Using data from the National Epidemiologic Survey on Alcohol and Related Conditions, this study examines the prevalence of withdrawal symptoms among inhalant users. Prevalence of inhalant withdrawal symptoms for inhalants was also compared with the prevalence of cocaine withdrawal symptoms to help determine the presence of an inhalant withdrawal syndrome.

Results: Approximately $47.8 \%$ of persons who met criteria for inhalant dependence reported experiencing three or more inhalant-related withdrawal symptoms that were clinically significant. Among those with inhalant dependence, almost half of the withdrawal symptoms were as common as the corresponding withdrawal symptoms experienced by persons with cocaine dependence. Furthermore, the percentage of persons with inhalant dependence reporting clinically significant inhalant withdrawal symptoms was almost equal to the percentage of persons with cocaine dependence reporting clinically significant cocaine withdrawal symptoms.

Conclusions: These data provide evidence for an inhalant-related withdrawal syndrome among persons with inhalant dependence. Revisions to DSM-IV should consider including inhalant withdrawal as a diagnostic criterion for this disorder.

Keywords: Inhalants, volatile solvents, withdrawal, inhalant use disorders

\section{Introduction}

Due to high risk for morbidity and mortality, inhalants are among the most dangerous forms of substance use, ${ }^{1,2}$ however, very little research on inhalant use disorders exists. ${ }^{3}$ The general absence of knowledge is apparent, as reflected in the short description of inhalant use disorders in the Diagnostic and Statistical Manual, 4th edition (DSM-IV). ${ }^{3}$ In particular, DSM-IV has included virtually no information regarding prevalence, course, subtypes, comorbid medical and mental health conditions, and specific age, gender, and sociodemographic features.

Recent findings have raised questions about the validity of DSM-IV criteria for inhalant use disorders. ${ }^{4}$ One serious challenge to the validity of inhalant dependence is that inhalant withdrawal symptoms are not considered to be present among inhalant users and therefore not part of the diagnostic criteria for making a DSM-IV dependence diagnosis. ${ }^{3,5}$ This decision was based on expert consensus rather than empirical evidence. At present, the DSM-V substance use disorder workgroup has proposed 
including inhalant withdrawal as part of a unidimensional inhalant use disorder (see http://www.dsm5.org/). However, this decision is not grounded in evidence. In fact, at the time of preparing this report, the DSM-V workgroup provides only a single citation in the rationale for proposed changes to inhalant use disorders specific to withdrawal. This citation is from alcohol research. ${ }^{6}$ As shown in a recent review, existing data from human and animal studies have suggested the presence of an inhalant withdrawal syndrome, especially among frequent or heavy inhalant users. ${ }^{5}$ However, to date, no study has systematically examined inhalant withdrawal among human subjects. Data from a large national sample could provide some evidence to guide the DSM-V workgroup.

A stronger knowledge base about inhalant-related symptoms is needed, as establishing the presence or absence of an inhalant withdrawal syndrome is critical to advancing the clinical assessment and treatment for inhalant use disorders. Valid diagnostic criteria are also necessary to ensure proper diagnosis of inhalant users and facilitate their timely access to treatment. Therefore, the purpose of this study was to examine the prevalence of withdrawal symptoms among inhalant users, and determine whether the data would support the presence of a withdrawal syndrome among inhalant-dependent users. The study sample was drawn from a nationally representative sample of inhalant users, a large national study that is presently known to contain the most comprehensive set of inhalant-specific withdrawal symptoms. Currently, no standards exist that indicate the extent to which withdrawal symptoms must occur for a given class of substances in order for withdrawal to be considered a reliable and valid indicator of substance dependence. Thus, to help contextualize the findings we compared the prevalence of inhalant withdrawal symptoms among persons who met DSM-IV criteria for inhalant dependence with the prevalence of cocaine withdrawal symptoms among persons who met DSM-IV criteria for cocaine dependence. We selected cocaine as a comparison group, given that the literature has clearly established a withdrawal syndrome for this substance. ${ }^{7}$ It should be noted that cannabis was not selected as a comparison group, as cannabis withdrawal is not recognized in DSM-IV because of doubts regarding its clinical significance at the time that DSM-IV was prepared. ${ }^{8}$ Nicotine was not used as a comparison, as the mechanisms and consequences of nicotine withdrawal are likely to exhibit fundamental differences. This is the first study to systematically examine inhalant withdrawal symptoms among humans, which fills a critical gap in inhalant research.

\section{Material and methods}

\section{Subjects, sampling, and interviews}

This study used data from the 2001-2002 National Epidemiologic Survey on Alcohol and Related Conditions (NESARC), a representative sample of 43,093 US adults, which gathered information on substance use and comorbid conditions from individuals living in households and group settings. ${ }^{9}$ This research was conducted with necessary permissions and complies with Institutional Review Board standards with respect to secondary data analysis. NESARC utilized a multistage sampling design and had an overall response rate of $81 \%$. Data were weighted at the individual and household levels and adjusted for oversampling, nonresponse, and to be representative of the US population vis-à-vis the 2000 census. US Census Bureau workers administered the Alcohol Use Disorders and Associated Disabilities Interview Schedule (AUDADIS-IV), a structured interview designed for administration by lay interviewers. AUDADIS-IV assesses DSM-IV mental health and substance use disorders with good reliability. ${ }^{9,10}$

\section{Measurement}

Inhalant use and inhalant use disorder

Inhalant users were identified in the overall sample with the following question: "Have you ever used inhalants or solvents; for example, amyl nitrite, nitrous oxide, glue, toluene, or gasoline?" An introductory statement preceding the inhalant question informed respondents that they were to report inhalants used on their own, without a doctor's prescription, and for the purposes of getting high, enjoying themselves, relaxing, feeling better, feeling more alert, quieting their nerves, or to see how they would work. Inhalant abuse was assigned to inhalant users who met DSM-IV inhalant abuse criteria but who did not meet inhalant dependence criteria; inhalant dependence was assigned to subjects who met DSM-IV inhalant dependence criteria. ${ }^{3}$

\section{Inhalant withdrawal symptoms}

The AUDADIS-IV contained a structured assessment of 18 substance-specific withdrawal symptoms for all substance classes. As described in Hasin et al, these questions addressed symptoms after cessation of use (ie, morning after or within the first few days of use). ${ }^{11}$ Inhalant-specific withdrawal symptoms are summarized in Table 1. The clinical significance of withdrawal was defined as the presence of two or more inhalant withdrawal symptoms and significant distress or impairment in social, occupational, or other life domains. Because the number of withdrawal symptoms required for meeting 
Table I Prevalence of withdrawal symptoms among persons with DSM-IV inhalant use disorders and cocaine dependence

\begin{tabular}{|c|c|c|c|c|c|c|}
\hline \multirow{2}{*}{$\begin{array}{l}\text { Withdrawal } \\
\text { symptom }\end{array}$} & \multirow{2}{*}{$\begin{array}{l}\text { Lifetime } \\
\text { inhalant use } \\
n=644 \\
\% \text { (SE) }\end{array}$} & \multicolumn{4}{|c|}{ DSM-IV inhalant use disorder classification } & \multirow{2}{*}{$\begin{array}{l}\text { Cocaine } \\
\text { dependence } \\
n=390 \\
\% \text { (SE) }\end{array}$} \\
\hline & & $\begin{array}{l}\text { No disorder } \\
\mathbf{n}=\mathbf{5 2 6} \\
\% \text { (SE) }\end{array}$ & $\begin{array}{l}\text { Inhalant } \\
\text { abuse }^{a} \\
n=I I 7 \\
\% \text { (SE) }\end{array}$ & $\begin{array}{l}\text { Inhalant } \\
\text { dependence }^{\mathrm{b}} \\
\mathbf{n}=\mathbf{2} \text { I } \\
\%(\mathrm{SE}) \\
\end{array}$ & $\begin{array}{l}\chi^{2} \text { test with } \\
\text { design-based } \\
\text { corrected } F(P)^{c}\end{array}$ & \\
\hline Hypersomnia & $4.4(0.98)$ & $1.7(0.63)$ & $9.4(2.79)$ & $63.6(13.9)$ & $62.9(<0.001)$ & $57.4(3.4 I)$ \\
\hline Feeling weak and tired & $7.9(1.14)$ & $4.0(0.99)$ & $20.0(4.26)$ & $55.4(15.16)$ & $28.5(0.001)$ & $77.3(2.7)$ \\
\hline Depressed mood & $4.9(0.97)$ & $1.3(0.53)$ & $17.2(4.42)^{\mathrm{e}}$ & $42.2(15.91)^{e}$ & $31.6(<0.001)$ & $73.5(3.16)$ \\
\hline $\begin{array}{l}\text { Sweating or fast } \\
\text { heart beat }\end{array}$ & $5.5(1.05)$ & $2.5(0.7 \mathrm{I})$ & $14.5(4.16)^{e}$ & $45.0(16.04)^{e}$ & $24.5(<0.00 \mathrm{I})$ & $70.0(3.15)$ \\
\hline Nausea & $4.8(1.07)$ & $2.1(0.74)$ & $12.2(3.52)$ & $46.0(15.95)$ & $28.3(<0.001)$ & $31.8(3.01)$ \\
\hline Yawning & $5.2(1.08)$ & $2.4(0.87)$ & $13.7(4.34)$ & $39.0(16.64)$ & $15.9(<0.00 \mid)$ & $35.8(3.27)$ \\
\hline Runny eyes and nose & $2.6(0.62)$ & I.0 (0.46) & $5.9(2.21)$ & $33.0(16.16)$ & $22.1(<0.001)$ & $59.9(2.89)$ \\
\hline $\begin{array}{l}\text { Eat more or gain } \\
\text { weight }\end{array}$ & $0.2(0.12)$ & $0.1(0.13)$ & $0(0.00)$ & $4.8(3.04)$ & $N A^{f}$ & I8.4 (2.3I) \\
\hline Anxiety & $5.4(1.07)$ & I.8 (0.73) & $17.4(4.53)^{\mathrm{e}}$ & $41.8(15.89)^{\mathrm{e}}$ & $25.4(<0.001)$ & $77.2(2.53)$ \\
\hline Muscle aches & $2.9(0.82)$ & $2.0(0.73)$ & $6.0(3.38)^{\mathrm{e}}$ & $11.0(6.30)^{\mathrm{e}}$ & $N A^{f}$ & II.4 (I.8I) \\
\hline Fever & $1.9(0.58)$ & $0.3(0.26)$ & $5.2(2.28)$ & $33.9(17.21)$ & $N A^{f}$ & II.4 (I.8I) \\
\hline Restlessness & $3.1(0.80)$ & I.8 (0.78) & $6.8(2.53)$ & $21.6(12.14)$ & $N A^{f}$ & $66.7(3.05)$ \\
\hline $\begin{array}{l}\text { Psychomotor } \\
\text { retardation }\end{array}$ & $3.7(0.8 \mathrm{I})$ & $2.2(0.70)$ & $8.2(3.14)^{\mathrm{e}}$ & $27.1(16.27)^{\mathrm{e}}$ & $9.1(<0.001)$ & $24.3(2.56)$ \\
\hline Sweating & $2.4(0.80)$ & $0.9(0.50)$ & $4.9(2.09)$ & $38.0(16.11)$ & $N A^{f}$ & $53.14(3.17)$ \\
\hline Vivid dreams & $3.1(0.82)$ & I.5 (0.7I) & $10.1(3.36)^{\mathrm{e}}$ & $7.7(4.35)^{e}$ & II.3 (<0.00I) & $34.4(2.82)$ \\
\hline Hallucination & $3.0(0.69)$ & I.3 (0.55) & 6.7 (2.39) & $35.3(16.27)$ & $21.0(<0.001)$ & $30.5(2.5 \mathrm{I})$ \\
\hline Tremors & $4.0(0.89)$ & I.8 (0.76) & 9.8 (3.09) & $37.8(16.7)$ & $17.6(<0.001)$ & $52.1(3.15)$ \\
\hline Insomnia & $2.1(0.58)$ & $0.5(0.30)$ & $6.3(2.40)$ & $28.6(16.16)$ & $N A^{f}$ & $61.0(2.98)$ \\
\hline Seizure & $0.5(0.28)$ & $0(0.00)$ & $2.5(1.59)$ & $2.4(2.48)$ & $N A^{f}$ & $5.0(1.17)$ \\
\hline Bad headaches & $7.3(1.25)$ & $3.8(1.06)$ & $20.3(4.70)^{\mathrm{e}}$ & $30.9(16.11)^{e}$ & $\mid 4.3(<0.00 \mathrm{I})$ & $4 I .6(2.7 I)$ \\
\hline $\begin{array}{l}\text { Any withdrawal } \\
\text { symptom }\end{array}$ & $19.0(1.89)$ & $12.2(1.78)$ & $43.6(5.32)$ & $77.3(12.60)$ & $34.8(<0.00 \mathrm{I})$ & $98.9(0.57)$ \\
\hline
\end{tabular}

Notes: All prevalence estimates of withdrawal symptoms are specific to the indicated drug (ie, inhalants or cocaine). ${ }^{\mathrm{a}}$ without a lifetime history of inhalant dependence; ${ }^{b}$ with or without a lifetime history of abuse; ${ }^{c}$ pair-wise comparisons were conducted when global test was statistically significant; ${ }^{d}$ withdrawal symptoms among cocaine dependence are reported for comparative purposes, but statistical comparisons were not made with inhalant use disorders because of nonmutually exclusive grouping; eall pair-wise comparison among inhalant use disorder groups were statistically significant except groups with this super-script; ${ }^{\text {fnot }}$ applicable due to cell counts $<5$.

Abbreviations: DSM-IV, Diagnostic and Statistical Manual, 4th edition; SE, standard error.

the clinical significance criterion varies by drug (eg, opiate withdrawal requires three withdrawal symptoms, and cocaine withdrawal requires two) and it has not yet been established for inhalant dependence, we also created a variable indicating the presence of three or more inhalant withdrawal symptoms (for those who had two or more symptoms that were clinically significant, as queried by the AUDADIS-IV). Lastly, the DSM-IV withdrawal criterion was calculated from the AUDADIS-IV inhalant withdrawal symptoms in NESARC, based on the presence of either 1) clinically significant withdrawal or 2) the use of the same or a similar substance to avoid or alleviate the withdrawal symptoms. ${ }^{3,11}$

\section{Analysis}

Analyses were conducted using Stata version $11 .{ }^{12}$ Stata implements a Taylor series linearization to adjust standard errors of estimates for complex survey sampling design effects involving clustered data. Percentages for each abuse and dependence criterion, withdrawal symptom, and other withdrawal characteristics were computed for all lifetime inhalant users. These percentages were also reported for each mutually excluded DSM-IV classification of inhalant use disorder (ie, no disorder, abuse, dependence). We also reported the prevalence of cocaine withdrawal symptoms and other characteristics among persons with cocaine dependence for comparative purposes. However, given the high rate of drug-related comorbidities among inhalant users ${ }^{13}$ statistical comparisons between respondents with inhalant dependence and cocaine dependence could not be performed. More specifically, mutually exclusive groups could not be established while achieving minimum cell counts for statistical comparisons.

\section{Results}

\section{Characteristics of inhalant users}

Overall, there were 664 inhalant users in the NESARC (unweighted). Approximately $74.2 \%$ of lifetime inhalant 
users were male and $82.2 \%$ were White. The majority was unmarried, and a substantial minority had a high school education or less. Only a small percentage of inhalant users had a DSM-IV drug use or alcohol use disorder that preceded inhalant use (7.8\% and 9.8\%). Approximately 1 in $11(9.3 \%)$ inhalant users had developed a DSM-IV mood and/or anxiety disorder prior to initiating inhalant use. Approximately one-third of the sample met criteria for conduct disorder or antisocial personality disorder. Approximately 22.2\% had a family history of behavior problems, and 50.5\% had a family history of substance use problems. The mean age of onset of inhalant use was 17.5 years $(95 \% \mathrm{CI}=16.0-17.9)$, range of 5-44 years. Additional demographic characteristics for inhalant users in NESARC are described elsewhere. ${ }^{14}$

\section{Summary of DSM-IV diagnostic criterion for inhalant use disorders}

The prevalence of DSM-IV inhalant use disorder criteria among the full sample of inhalant users was low; that is, less than $13 \%$ for any given criterion, with the exception of a desire to or unsuccessful efforts to control (30.4\%) (see Table 2). However, a clear graded relationship among the diagnostic groups is present. By graded relation, we mean that those without any disorder have the lowest prevalence, followed by persons who met lifetime criteria for abuse, and then dependence.

Of the 11 inhalant abuse and dependence criteria, withdrawal was ranked as the 7 th most common criteria among all inhalant users. For those without an inhalant disorder, the ranking of withdrawal was 5 th (inhalant abuse, 7 th; inhalant dependence, 6th). Among persons with inhalant dependence, the majority (53.4\%) experienced withdrawal. Among the seven inhalant dependence criteria, withdrawal ranked as the 6 th most common. The least common was using larger amounts/longer periods (38.4\%).

\section{Comparison of inhalant withdrawal symptoms}

As described in Table 1, approximately one in five inhalant users reporting lifetime inhalant use experienced at least one type of inhalant withdrawal symptom. A graded relationship across the DSM-IV diagnostic categories was observed for any lifetime history of inhalant withdrawal symptoms. More specifically, $12.2 \%$ of inhalant users (no disorder) reported any withdrawal symptom, whereas the percentages for those who met criteria for inhalant abuse $(43.6 \%)$ or dependence $(77.3 \%)$ were significantly higher $(P<0.001)$. A graded relationship across the DSM-IV diagnostic categories was also observed for each unique withdrawal symptom, with the exception of vivid dreams. The differences across groups reached significance for each group that allowed statistical comparisons, with the exception of muscle aches. Due to low cell counts and multiple comparisons, it is important that the differences are interpreted cautiously.

The most commonly reported withdrawal symptoms among persons with inhalant dependence were hypersomnia (63.6\%), feeling tired (55.4\%), and nausea (46.0\%). The least common were seizures $(2.4 \%)$, eating more or gaining weight $(4.8 \%)$, and vivid dreams (7.7\%). For comparative purposes,

Table 2 Prevalence of DSM-IV abuse and dependence criteria among persons with lifetime inhalant use

\begin{tabular}{|c|c|c|c|c|}
\hline \multirow[t]{2}{*}{ Diagnostic criteria } & \multirow{2}{*}{$\begin{array}{l}\text { Lifetime } \\
\text { inhalant use } \\
n=644 \\
\%(S E)\end{array}$} & \multicolumn{3}{|c|}{ DSM-IV inhalant use disorder classification } \\
\hline & & $\begin{array}{l}\text { No disorder } \\
\mathbf{n}=\mathbf{5 2 6} \\
\% \text { (SE) }\end{array}$ & $\begin{array}{l}\text { Inhalant abuse }{ }^{a} \\
n=117 \\
\%(S E)\end{array}$ & $\begin{array}{l}\begin{array}{l}\text { Inhalant } \\
\text { dependence }\end{array} \\
\mathbf{n}=\mathbf{2} \text { I } \\
\% \text { (SE) }\end{array}$ \\
\hline AI Failure to fulfill role obligations & $7.8(1.34)$ & $N A^{c}$ & $35.6(5.19)$ & $75.5(10.52)$ \\
\hline A2 Hazardous use & $12.5(1.75)$ & $N A^{c}$ & $62.9(5.22)$ & $65.1(14.65)$ \\
\hline A3 Legal problems & $1.0(0.37)$ & $N A^{c}$ & $2.8(1.46)$ & $20.9(I I .6 I)$ \\
\hline A4 Use despite social or interpersonal problems & $11.6(1.48)$ & $N A^{c}$ & $58.9(5.50)$ & $65.5(16.24)$ \\
\hline DI Tolerance & $3.7(0.89)$ & $\mathrm{I} .0(0.42)$ & $9.32(2.67)$ & $56.7(14.67)$ \\
\hline D2 Withdrawal & $5.6(1.12)$ & $1.6(0.65)$ & I8.I (4.74) & $53.4(15.33)$ \\
\hline D3 Larger amounts/longer periods & $4.7(0.85)$ & $1.7(0.66)$ & I $4.7(3.44)$ & $38.4(\mid 3.62)$ \\
\hline D4 Desire to or unsuccessful efforts to control & $30.4(2.27)$ & $24.7(2.48)$ & $49.6(5.35)$ & $87.2(11.72)$ \\
\hline D5 Great deal of time spent & $7.3(1.12)$ & $2.6(0.87)$ & $19.9(3.92)$ & $79.1(11.65)$ \\
\hline D6 Activities given up or reduced & $4.1(0.82)$ & $0.6(0.37)$ & $11.6(3.23)$ & $70.1(12.37)$ \\
\hline $\begin{array}{l}\text { D7 Continued use despite psychological or } \\
\text { physical problems }\end{array}$ & $7.0(1.20)$ & $2.1(0.74)$ & $21.9(4.32)$ & $68.5(13.90)$ \\
\hline
\end{tabular}

Notes: ${ }^{a}$ without a lifetime history of inhalant dependence; ${ }^{b}$ with or without a lifetime history of abuse; cnot applicable, as endorsement of any of these criterion qualifies the respondent for an inhalant abuse disorder.

Abbreviations: DSM-IV, Diagnostic and Statistical Manual, 4th edition; SE, standard error. 
the percentages of cocaine withdrawal symptoms among persons with cocaine dependence are presented in Table 1. However, formal statistical tests between the inhalant and cocaine groups were not possible, given that the groups are not mutually exclusive. A visual inspection of the percentages suggested that 6 of the 21 withdrawal symptoms among inhalant-dependent individuals occurred as prevalent or more prevalent than the corresponding rates among cocaine-dependent individuals. Prior research has not clearly defined how many drug users must exhibit a given withdrawal symptom for it to be considered frequently occurring. If the threshold is set at $30 \%, 13$ unique inhalant withdrawal symptoms would be considered frequently occurring among persons with inhalant dependence, and 15 unique cocaine withdrawal symptoms would be considered frequently occurring among persons with cocaine dependence. If the threshold is set at 50\%, two inhalant withdrawal symptoms and 10 cocaine withdrawal symptoms would be considered frequently occurring.

\section{Comparison of withdrawal summary characteristics}

Summary variables revealed that individual withdrawal symptoms and an analog of the DSM-IV withdrawal criteria were experienced most frequently among those with inhalant dependence (see Table 3). Persons without a disorder exhibited the lowest frequency of withdrawal symptoms, followed by persons with abuse. The mean number of inhalant withdrawal symptoms among those with inhalant dependence was 6.5. A regression model of all inhalant users revealed that the mean number of withdrawal symptoms was significantly different across the diagnostic groups, and a series of pairwise comparisons showed differences across the groups $(P<0.05)$. Again, these tests must be interpreted conservatively due to low cell counts and multiple comparisons. Based on a visual inspection, the mean number of withdrawal symptoms for those with inhalant dependence was slightly lower than the mean number of withdrawal symptoms among those with cocaine dependence (9.2). The percentage of persons with inhalant dependence who reported two or more withdrawal symptoms that were clinically significant $(49.2 \%)$ was close to the percentage of those with cocaine dependence $(48.9 \%)$. The percentages remained close when the threshold was increased to three, with at least two being clinically significant (inhalant dependence $=47.8 \%$; cocaine dependence $=48.7 \%$ ). However, almost half of persons with cocaine dependence (48.0\%) used substances to avoid bad after effects, whereas only $10.3 \%$ of persons with inhalant dependence did. Again, it should be noted that comparisons between inhalant and cocaine dependence were based on visual inspections. Lastly, the DSM-IV criterion (experiencing more than two withdrawal symptoms that cause clinically significant distress, or using the same or a similar substance to avoid withdrawal) was experienced significantly more often in those with inhalant dependence (53.4\%) as compared with those with inhalant abuse $(18.1 \%)$ or use $(1.6 \%)$. The withdrawal criterion was experienced slightly more often in those with cocaine dependence $(69.8 \%)$.

\section{Conclusion}

\section{Reprise of findings}

To date, the majority of research on inhalant withdrawal among human subjects has utilized a case study method. ${ }^{5}$ This line of research provided an argument for the presence of a withdrawal syndrome among heavy users of inhalants. In the current study, we provide empirical support for inhalant withdrawal syndrome in a community sample of inhalant users who met DSM-IV criteria for inhalant dependence. Overall, these data show a high prevalence of withdrawal symptoms among inhalant-dependent inhalant users. Among those with inhalant dependence, almost half of the withdrawal symptoms were as common as the corresponding withdrawal symptoms experienced by persons with cocaine dependence. Furthermore, the percentage of persons with inhalant dependence reporting clinically significant inhalant withdrawal symptoms was almost equal to the percentage of persons with cocaine dependence reporting clinically significant cocaine withdrawal symptoms. These data provide initial evidence for the existence of an inhalant withdrawal syndrome. That is, over half the persons who met criteria for dependence also experienced clinically significant withdrawal, providing empirical evidence for the addition of inhalant withdrawal as a diagnostic criterion in future revisions of the DSM.

A particularly interesting finding was the difference in manifestation of inhalant withdrawal symptoms and cocaine withdrawal symptoms. As previously indicated, almost half of persons with cocaine dependence use cocaine to avoid bad after effects, whereas only $10.3 \%$ of persons with inhalant dependence did. In addition, the symptom "eat more or gain weight" was reported by approximately one in five of those with cocaine dependence, but only one in 20 of those with inhalant dependence. Assuming the existence of an inhalant withdrawal syndrome, these data suggest a clear difference in manifestation with cocaine and possibly other substances. This suggests further work needs to be done on the assessment and construct validity of inhalant withdrawal. Ideally, it would be useful to know which symptoms are 


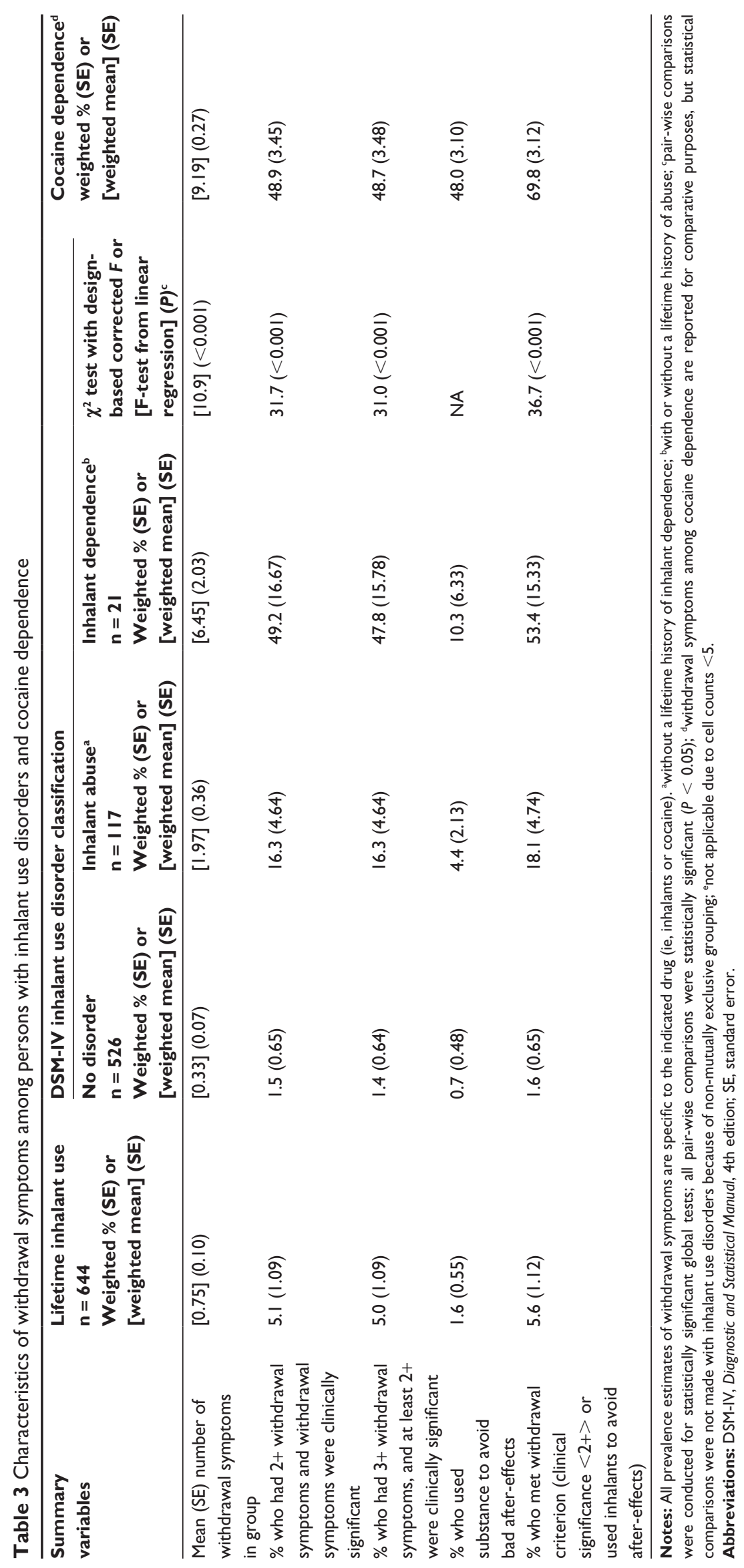


most common among inhalant users, especially for different types of inhalants.

\section{Study limitations}

It is important to consider these findings and recommendations in context of the study limitations. Most notably, the unweighted sample size of those who met DSM-IV criteria for dependence was small $(n=21)$, which makes these estimates less precise than estimates for other substances (eg, cocaine). However, the list of withdrawal symptoms is comprehensive and represents a substantial improvement over the case study approach. ${ }^{5}$ Another consideration is the issue of comorbid substance use disorders among inhalant users. Prior research revealed that the lifetime prevalence of comorbid substance use disorders among NESARC inhalant users was $96 \% .{ }^{14}$ Given the high rate of comorbidity, it is nearly impossible to define a sample of persons who use inhalants only. A subset of poly-drug users may not be able to effectively discern whether inhalant symptoms are due to only inhalants or possibly other substances. For the same reasons, it would be difficult to identify characteristics of inhalantonly users in a purely clinical sample. However, in a study of cannabis withdrawal, Hasin et al found that the prevalence of each withdrawal symptom was very close in a frequent cannabis user group (use of cannabis three or more days a week) with comorbidity and a cannabis-only subset (users who never had five or more drinks on a single occasion and never used other drugs three or more days a week). ${ }^{11}$ Further investigation is necessary to establish the extent to which the inhalant withdrawal symptoms are specific to inhalant use. It would be reasonable to focus initially on the most commonly occurring withdrawal symptoms observed in this national survey (ie, hypersomnia, depressed mood, feeling weak and tired, and nausea). For those with comorbid drug dependence disorders, it would also be helpful to know whether these symptoms are strongly associated with withdrawal symptoms resulting from use of other drugs. This would help define symptom specificity.

The most complicated issues to be addressed relate to the within-group heterogeneity of inhalant use. Inhalants represent the only substance based on the mode of administration rather than specific agent or chemical property. ${ }^{15}$ Prior research has also revealed that inhalant users may use multiple inhalant products. For example, $\mathrm{Wu}$ and Ringwalt examined inhalant use among a nationally representative sample of adult inhalant users and found that $22.6 \%$ used two different types of inhalant products, and $15.5 \%$ used three or more inhalant products. ${ }^{16}$ In another study, using a large sample of adolescent inhalant users, Howard et al found that $47.9 \%$ of individuals reporting lifetime inhalant use had tried four or more inhalant products. ${ }^{17}$ This raises questions as to whether certain products are more likely to give rise to inhalant withdrawal symptoms than other products. Qualitative research that addresses the phenomenology of inhalant use can help us understand what other withdrawal symptoms might be unique to inhalants (between group differences), in addition to helping link specific withdrawal symptoms to specific inhalant products. This is a critical issue to address, as the physiological and psychological processes associated with the various inhalant products may be fundamentally different from each other. ${ }^{15}$

\section{Conclusions and future directions}

Overall, this study found support for the presence of withdrawal symptoms and an inhalant withdrawal syndrome among persons who meet criteria for inhalant dependence. Including withdrawal as a diagnostic criterion for inhalant use disorders in the next revision of the DSM is warranted given the prevalence and clinical significance of these symptoms. Future research needs to improve on screening of inhalant use in general and the assessment of withdrawal symptoms specifically. This may help to reveal which withdrawal symptoms are specific to inhalants, or whether the symptoms manifest in conjunction with other drugs, and to inform the designs of effective treatment approaches specific to addressing inhalant dependence. Nonetheless, when inhalant users are identified in the addiction treatment setting, medical management of their withdrawal symptoms is an important aspect in addressing each of the DSM characteristics.

\section{Acknowledgments}

NESARC was funded by the National Institute on Alcohol Abuse and Alcoholism with additional support provided by the National Institute on Drug Abuse. Data analysis and writing of this article was supported by grants DA027832, DA019623, DA019901, and DA027503, from the National Institute on Drug Abuse. The National Institute on Drug Abuse had no further role in the design or conduct of this study; collection, management, analysis, and interpretation of the data; or preparation, review, or approval of the manuscript. All authors participated in the conceptualization of the study. The first and second authors conducted statistical analyses, and all authors participated in the manuscript preparation.

\section{Disclosure}

None of the authors have a financial interest or relationship with an individual or organizational entity that constitutes a 
conflict of interest with regard to the subject matter of this manuscript.

\section{References}

1. Dinwiddie SH. Abuse of inhalants: a review. Addiction. 1994;89(8): 925-939.

2. Dinwiddie SH. Psychological and psychiatric consequences of inhalants. In: Tarter RE, Ammerman RT, Ott PJ, editors. Handbook of Substance Abuse: Neurobehavioral Pharmacology. New York: Plenum; 1998.

3. American Psychiatric Association. Diagnostic and Statistical Manual of Mental Disorders. 4th ed. Washington, DC: American Psychiatric Association; 2000.

4. Ridenour TA, Bray BC, Cottler LB. Reliability of use, abuse, and dependence of four types of inhalants in adolescents and young adults. Drug Alcohol Depend. 2007;91(1):40-49.

5. Perron BE, Howard MO, Vaughn MG, et al. Inhalant withdrawal as a clinically significant feature of inhalant dependence disorder. Medical Hypotheses. 2009;73(6):935-937.

6. De Bruijn C, van den Brink W, de Graaf R, et al. The craving withdrawal model for alcoholism: towards the DSM-V. Improving the discriminate validity of alcohol use disorder diagnosis. Alcohol Alcohol. 2005; 40(4):314-322.

7. Kampman KM, Alterman AI, Volpicelli JR, et al. Cocaine withdrawal symptoms and initial urine toxicology results predict treatment attrition in outpatient cocaine dependence treatment. Psychol Addict Behav. 2001; 15(1):52-59.

8. Levin KH, Copersino ML, Heishman SJ, et al. Cannabis withdrawal symptoms in non-treatment-seeking adult cannabis smokers. Drug Alcohol Depend. 2010;111(1-2):120-127.
9. Grant BF, Dawson DA, Stinson FS, et al. The Alcohol Use Disorder and Associated Disabilities Interview Schedule-IV (AUDADIS-IV): Reliability of alcohol consumption, tobacco use, family history of depression and psychiatric diagnostic modules in a general population sample. Drug Alcohol Depend. 2003;71(1):7-16.

10. Grant BF, Harford T, Dawson DA, et al. The Alcohol Use Disorder and Associated Disabilities Interview schedule (AUDADIS): reliability of alcohol and drug modules in a general population sample. Drug Alcohol Depend. 1995;39(1):37-44.

11. Hasin DS, Keyes KM, Alderson D, et al. Cannabis withdrawal in the United States: results from NESARC. J Clin Psychiatry. 2008;69(9): 1354-1363.

12. StataCorp. Stata Statistical Software: Release 11. College Station, TX: StataCorp, LP; 2009.

13. Wu L-T, Howard MO, Pilowsky DJ. Substance use disorders among inhalant users: results from the National Epidemiologic Survey on alcohol and related conditions. Addict Behav. 2008;33(7):968-973.

14. Perron BE, Howard MO, Maitra S, et al. Prevalence, timing, and predictors of transitions from inhalant use to inhalant use disorders. Drug Alcohol Depend. 2009;100(3):277-284.

15. Balster RL, Cruz SL, Howard MO, et al. Classification of abused inhalants. Addiction. 2009;104(6):878-882.

16. Wu L-T, Ringwalt CL. Inhalant use and disorders among adults in the United States. Drug Alcohol Depend. 2006;85(1):1-11.

17. Howard MO, Balster RL, Cottler LB, et al. Inhalant use among incarcerated adolescents in the United States: prevalence, characteristics, and correlates of use. Drug Alcohol Depend. 2008;93(3):197-209.
Substance Abuse and Rehabilitation

\section{Publish your work in this journal}

Substance Abuse and Rehabilitation is an international, peer-reviewed, open access journal publishing original research, case reports, editorials, reviews and commentaries on all areas of addiction and substance abuse and options for treatment and rehabilitation. The manuscript management system is completely online and includes a very quick and

\section{Dovepress}

fair peer-review system. Visit http://www.dovepress.com/testimonials. php to read real quotes from published authors. 